\title{
Editorial
}

\section{Harmony Search and Nature-Inspired Algorithms for Engineering Optimization}

\author{
Zong Woo Geem, ${ }^{1}$ Xin-She Yang, ${ }^{2}$ and Chung-Li Tseng ${ }^{3}$ \\ ${ }^{1}$ Department of Energy and Information Technology, Gachon University, Seongnam 461-701, Republic of Korea \\ ${ }^{2}$ Department of Design Engineering and Mathematics, Middlesex University, London NW4 4BT, UK \\ ${ }^{3}$ The Australian School of Business, The University of New South Wales, Sydney, NSW 2052, Australia \\ Correspondence should be addressed to Zong Woo Geem; geem@gachon.ac.kr
}

Received 7 November 2013; Accepted 7 November 2013

Copyright (c) 2013 Zong Woo Geem et al. This is an open access article distributed under the Creative Commons Attribution License, which permits unrestricted use, distribution, and reproduction in any medium, provided the original work is properly cited.

Since the emergence of swarm intelligence in the 1990s, especially the appearance of ant colony optimization and particle swarm optimization, nature-inspired algorithms started to mushroom [1-7]. In the last two decades, new optimization algorithms have become popular, and the algorithms such as harmony search $[1,2]$ and firefly algorithms [5] have shown to be superior and efficient. Significant progress has been made in a wide range of nature-inspired algorithms and their applications. Metaheuristics and swarm intelligence are becoming more popular for design optimization [3-5].

Most of these algorithms belong to evolutionary computation in general, and they have been developed and inspired from natural phenomenon. However, not all algorithms were inspired by nature. For example, harmony search, developed by Geem et al. in 2001, was inspired by the improvisation characteristics of a musician, and therefore, harmony search is a music-inspired algorithm $[1,2]$. However, a vast majority of algorithms have been developed by mimicking the characteristics of biological systems in nature. For example, the firefly algorithm was developed by Yang in 2009 [5] and was inspired by the flashing patterns of tropical fireflies, while the cuckoo search was developed by Yang and Deb in 2010, inspired by the brooding parasitism of some cuckoo species [6]. The diversity of these algorithms and their applications has permeated into almost every area of engineering and industry [3-5].

The aim of this special issue is to review the latest developments in nature-inspired algorithms and their applications. The call for papers was well received, leading to a high number of submissions. After a rigorous peer-review process, sixteen high-quality papers have been selected. These papers represent a snapshot of the relevant research progress in these areas. Ten of the papers published in this special issue are dedicated to harmony search (HS) and the others to other types of nature-inspired algorithms. M. A. Al-Betar et al. present a new variant of HS, called cellular harmony search, to solve a set of 25 test functions with promising results. S. S. Im et al. also propose using cellular harmony search for unconstrained optimization. D. Oliva et al. use harmony search to carry out multilevel thresholding segmentation in image processing. Z. W. Geem demonstrates a parametersetting-free version of harmony search and uses it to solve the economic dispatch of power plant operations. J. Fourie et al. carry out a comparative analysis of modern harmony search, providing new insights into the working mechanism of the method. R. Mallipeddi combines harmony search with differential evolution to enhance differential evolution by using parameter ensemble adaptation. In another paper, H.-Y. Yun et al. combine harmony search and ant colony optimization for traveling salesman problem. Furthermore, $\mathrm{S}$. Lee et al. determine pavement rehabilitation activities by a permutation algorithm within the framework of harmony search. In the other two applications using harmony search, $\mathrm{S}$. Lee et al. solve electroencephalography signal grouping, and D.-S. An et al. estimate parameters for traffic noise.

For those papers on nature-inspired algorithms, A. Gálvez and A. Iglesias use a firefly algorithm-based approach to do polynomial Bézier surface parameterization for 
complex surfaces. O. Baskan uses the cuckoo search to determine optimal link capacity expansions in road networks, while $\mathrm{H}$. Zheng and Y. Zhou develop a variant of cuckoo search, called a cooperative coevolutionary cuckoo search algorithm. They have solved 28 test problems and achieved better results. In addition, Ma et al. use the cuckoo search to estimate key parameters in photovoltaic models and obtain high accuracy in terms of a low root-meansquared-error value. For practical applications, J. Xie et al. use the bat algorithm to solve multiple runways aircraft landing problem, such as up to 500 aircrafts landing safely in a short time. S. Fong et al. use swarm search to select optimal feature set in high-dimensional data.

The papers published in this special issue give a glimpse of the current development and the active research in harmony search, firefly algorithm, cuckoo search, and bat algorithms as well as other algorithms. This special issue also reports new real-world applications, indicating that the applicability of these algorithms continues to expand. Obviously, this special issue can only represent a fraction of current research activities among the more diverse studies and applications.

Despite the success of nature-inspired algorithms, in our view, there are at least three challenges that require special attention of the researchers: large-scale problems, combinatorial optimization, and proper constraint handling.

Currently, most studies in the literature concern problems with a small or moderate number of design variables. It is rare to see a study dealing with hundreds of design variables. Recently, large-scale problems have started to be tackled by researchers (e.g., in $[3,4])$. However, more research activities and efforts are needed as real-world applications can become extremely complex with potentially millions of design variables.

The vast majority of the applications of evolutionary algorithms have focused on continuous optimization problems. There is another important class of challenging problems called combinatorial optimization, such as the well-known traveling salesman problem. Since combinatorial optimization problems can be NP-hard, nature-inspired algorithms are good candidates for tackling this type of problems. While there have been researchers attempting to address this issue, such as water distribution networks (one of which has 1E454 candidate solutions) and the traveling salesman problem $[3,4]$, for truly hard problems and large-scale ones, more systematic efforts and studies are demanded to provide better results in a more efficient way.

Another important issue is constraint handling, from the implementation point of view. Even if an efficient algorithm is correctly implemented, its performance can be hampered if the constraints of the problem are not properly handled. While some may view constraint handling as a separate topic, it is part of the integrated optimization process. If the solution of a real application generated by algorithms does not satisfy the constraints, the solution is infeasible and cannot be implemented. This highlights the importance of constrainthandling techniques in combination with optimization algorithms.

In this special issue, the selected papers only address a small subset of the vast number of optimization problems.
We hope this special issue will encourage and inspire more needed research activities in the near future.

\section{Acknowledgment}

We would like to thank those who participated in this special issue, including the contributing authors and reviewers.

\section{Zong Woo Geem \\ Xin-She Yang \\ Chung-Li Tseng}

\section{References}

[1] Z. W. Geem, J. H. Kim, and G. V. Loganathan, "A new heuristic optimization algorithm: harmony search," Simulation, vol. 76, no. 2, pp. 60-68, 2001.

[2] Z. W. Geem, Music-Inspired Harmony Search Algorithm, Springer, Heidelberg, Germany, 2009.

[3] Z. W. Geem, "Optimal cost design of water distribution networks using harmony search," Engineering Optimization, vol. 38, no. 3, pp. 259-280, 2006.

[4] A. Ouaarab, B. Ahiod, and X. S. Yang, "Discrete cuckoo search algorithm for the travelling salesman problem," Neural Computing and Applications, 2013.

[5] X.-S. Yang, "Firefly algorithms for multimodal optimization," in Stochastic Algorithms: Foundations and Applications, vol. 5792 of Lecture Notes in Computer Science, pp. 169-178, Springer, Berlin, Germany, 2009.

[6] X.-S. Yang and S. Deb, "Engineering optimisation by cuckoo search," International Journal of Mathematical Modelling and Numerical Optimisation, vol. 1, no. 4, pp. 330-343, 2010.

[7] X. S. Yang, M. Karamanoglu, and X. S. He, "Flower pollination algorithm: a novel approach for multiobjective optimization," Engineering Optimization, 2013. 


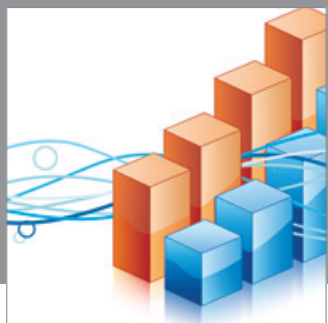

Advances in

Operations Research

mansans

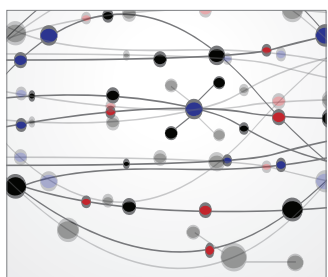

The Scientific World Journal
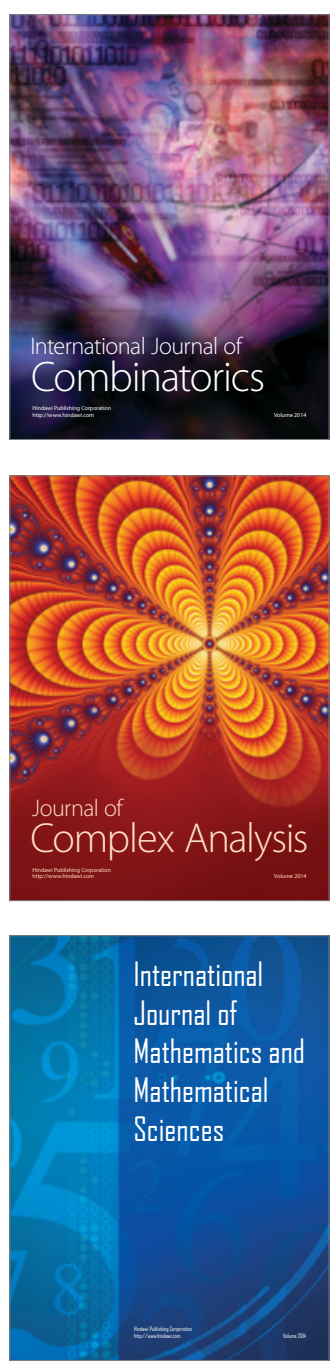
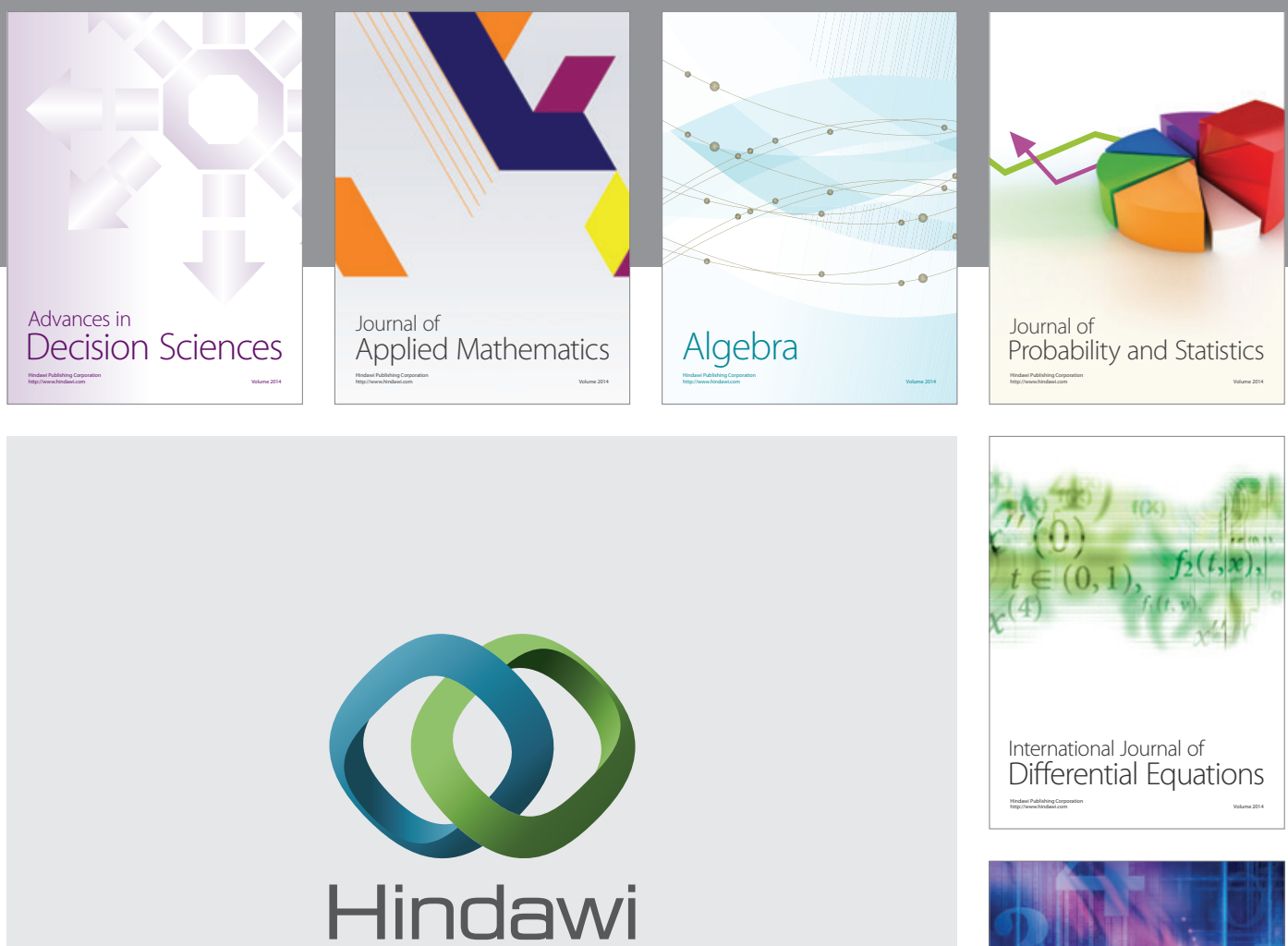

Submit your manuscripts at http://www.hindawi.com
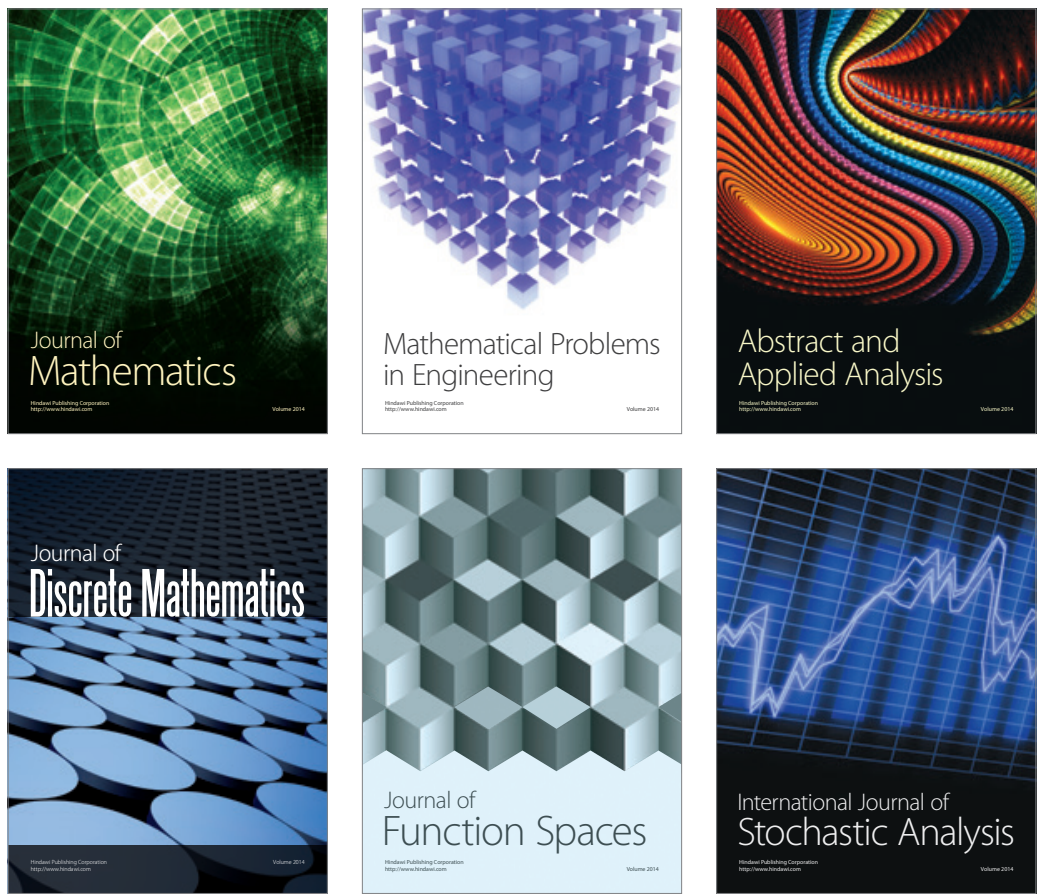

Journal of

Function Spaces

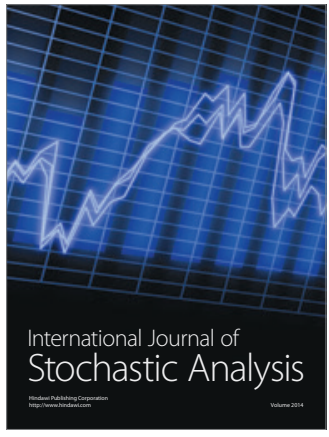

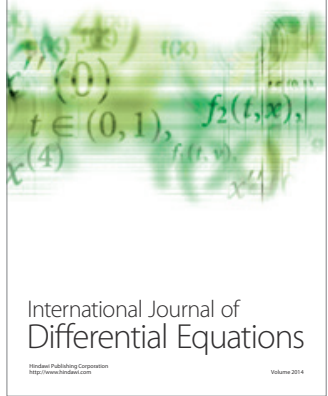
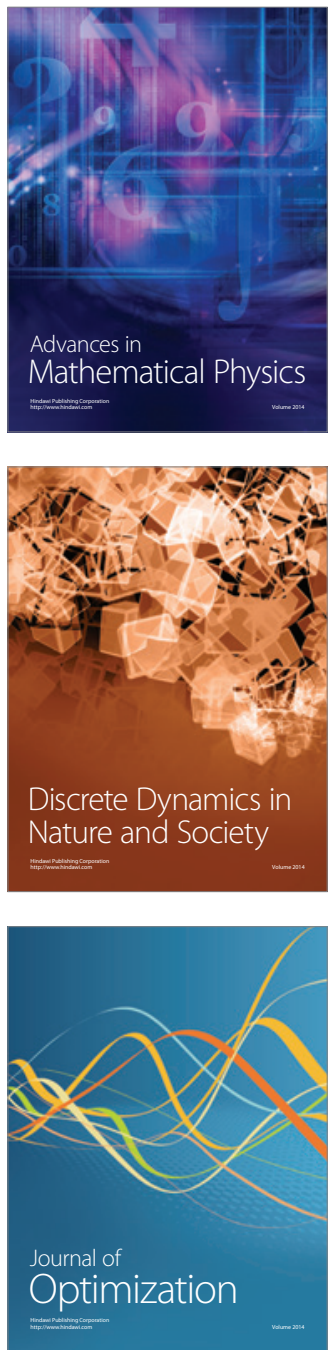